\title{
'Thinking you're old and frail': a qualitative study of frailty in older adults
}

\author{
KRYSTAL WARMOTH*†, IAIN A. LANG*†, CASSANDRA PHOENIX*, \\ CHARLES ABRAHAM $* \dagger$, MELISSA K. ANDREW $\ddagger$, \\ RUTH E. HUBBARD $\$$ and MARK TARRANT*+
}

\begin{abstract}
Many older adults experience what is clinically recognised as frailty but little is known about the perceptions of, and attitudes regarding, being frail. This qualitative study explored adults' perceptions of frailty and their beliefs concerning its progression and consequences. Twenty-nine participants aged 66-98 with varying degrees of frailty, residing either in their homes or institutional settings, participated in semistructured interviews. Verbatim transcripts were analysed using a Grounded Theory approach. Self-identifying as 'frail' was perceived by participants to be strongly related to their own levels of health and engagement in social and physical activity. Being labelled by others as 'old and frail' contributed to the development of a frailty identity by encouraging attitudinal and behavioural confirmation of it, including a loss of interest in participating in social and physical activities, poor physical health and increased stigmatisation. Using both individual and social context, different strategies were used to resist self-identification. The study provides insights into older adults' perceptions and attitudes regarding frailty, including the development of a frailty identity and its relationship with activity levels and health. The implications of these findings for future research and practice are discussed.
\end{abstract}

KEY WORDS - frailty, identity, perceptions of ageing, qualitative study.

\section{Introduction}

The term 'frailty' has been increasingly used to refer to the health conditions of many older adults (e.g. Borges and Menezes 2011; Heuberger $2011)$. In biomedical research, the term is used to classify physical aspects

* University of Exeter Medical School, Exeter, UK.

$\dagger$ Peninsula Collaboration for Leadership in Applied Health Research and Care, Exeter, UK.

\$ Division of Geriatric Medicine, Dalhousie University, Halifax, Canada.

$\S$ Centre for Research in Geriatric Medicine, University of Queensland, Brisbane, Australia. 


\section{Krystal Warmoth et al.}

of ageing. However, its conceptualisation is debated-as a physical syndrome caused by sarcopenia which can be measured by the Fried phenotype or the FRAIL scale (Morley, Perry and Miller 2002), or as a risk state which is secondary to accumulated deficits of illnesses, functional impairments, sensory impairments and symptoms (Clegg et al. 2013). It is estimated that between 25 and $5^{\circ}$ per cent of people who are 85 years or older experience what is clinically recognised as frailty (Clegg et al. 2013). However, older adults' understanding is not often acknowledged and reflected in these conceptualisations. The aim of this qualitative study was to gain a more detailed understanding of older adults' perceptions of frailty and their beliefs concerning its progression and consequences.

Definitions of frailty are important because they can guide treatment decisions in clinical contexts (Borges and Menezes 2011). However, it is also important to consider how older people themselves perceive frailty because perceptions regarding health can play an important role in determining the progression of illness (Petrie, Jago and Devcich 2007). To this end, the current research explored the discourses of frailty used by older people. Few studies have examined people's perceptions regarding being frail, but those that have highlight the complex and nuanced nature of this condition. For example, Dutch older adults in the study of Puts et al. (2009) described frailty both as a state of general physical impairment and disease, and as a condition with delineable social and psychological ill-effects. Participants highlighted a reduced ability to cope with their physical limitations, negative emotions (e.g. fear), impaired cognitive functioning and poor social interactions. Consistent with this multi-dimensional conceptualisation of frailty, older women in Grenier's (2005, 2006) research described frailty in relation to emotional responses and in the context of their functional limitations and physical changes. Other discourses of frailty describe not only an association with physical capacity but also one of powerlessness, dependency and cognitive decline, reflecting feared aspects of ageing (Grenier 2007).

The current research considered the interaction between people's beliefs about frailty with their general self-perceptions and identities. Theories of identity management in later life (e.g. Coleman 1999) indicate that identity is negotiated in response to life experiences and particular changes in personal circumstances. Fillit and Butler (2009) suggested physical impairment resulting from the loss of independence or control of one's body can lead people to self-identify as old. Predominantly negative, reflecting common old-age stereotypes (impaired, despondent or recluse; Hummert 2011), self-identifying as old can have implications for health and wellbeing. Indeed, people showing symptoms of frailty often concurrently report low levels of wellbeing, suggesting that defining oneself in terms of a 'frailty 
identity' (as frail) might play an important role in determining the health outcomes of older adults (Andrew, Fisk and Rockwood 2012). The present study considered the perceptions of the self and others with one's understanding of frailty.

In addition to exploring older people's perceptions and understandings of frailty, the current research explored whether frailty as an identity is something older people perceive as an inevitable consequence of ageing, or whether it is something actively managed or resisted. Suggesting that identity change is not necessarily an inevitable part of ageing, some studies show that older people play an active role in the construction of their own identities (Cotter and Gonzalez 2009). While recognising their physical symptoms of frailty, many older people in Grenier's (2006) study simultaneously rejected the frailty label and distinguished between 'being frail' and 'feeling frail'. 'Being frail' was viewed as an imposed medical or functioning classification, while 'feeling frail' reflected the emotional consequences of traumatic events (e.g. loss of a loved one), disability or physical impairment. Similar findings were reported by Puts and colleagues (Puts et al. 2009), whose participants did not identify as 'frail' even when they met the classification criteria on a standardised measure (Puts, Lips and Deeg 2005). Resisting a frailty identity may be one such negotiation strategy that some older people employ in order to maintain a positive self in response to age-related changes or ill-health experiences. Thus, by resisting the frailty identity, an 'identity crisis' may be avoided (Fillit and Butler 2009).

The above review indicates older adults' understanding and experience of frailty is nuanced, and not just a physical state related to ageing or physical weakness. It also suggests that self-identifying as frail, while potentially damaging to health and wellbeing, might not be an inevitable, or automatic, consequence of age-related change. However, while a distinction can be drawn between 'being frail' and 'feeling frail', very little is known about how older people make this distinction or how it relates to their health and wellbeing. The current study sought to explore these processes further. Specifically, the aim of the study was to examine qualitatively older adults' perceptions of frailty and their beliefs concerning its progression and health consequences.

\section{Methods}

A Grounded Theory approach was adopted because its methodology is a wellestablished and flexible approach to construct theory from data (Charmaz 2006). Using this approach, participants' own perspectives can be explored and an explanatory theory developed (Glaser and Strauss 1967). 


\section{Data collection}

Open purposive sampling (Strauss and Corbin 1998) was undertaken through a network of research contacts and community organisations (including Age UK, the local Senior Voice organisation, and patient and public involvement contacts at the first author's institution). These organisations and contacts acted as gatekeepers and were sent a recruitment poster with details about the study (e.g. project aims, eligibility criteria, time commitment). Respondents were included in the study if they were over the age of $6_{5}$ years and excluded if they were unable to consent independently (e.g. due to severe cognitive impairment). Posters were displayed in each gatekeeper's work environment and copies were distributed to interested individuals. Once eligibility had been confirmed, participants were provided with an information pack and asked to provide informed consent before being interviewed. In later interviews, recruitment to the study became more selective and employed theoretical sampling (a technique for seeking pertinent data from relevant sources to develop the emerging theory; Charmaz 2006). Data collection ended when no further theoretical insights were obtained from the data, or theoretical saturation was reached (Charmaz 2006). The study was approved by the first author's institutional ethics committee.

Semi-structured interviews were conducted in participants' homes or a private meeting room on the university campus of the first author. A topic guide was used to direct the interviews, which included key themes of health, daily life, experiences of getting older and participants' understanding of frailty. Participants were encouraged to use their own words to explain their experiences of ageing and attitudes towards frailty. Openended questions and probing for further clarification was used to facilitate this process as necessary. Recurring themes and emerging common issues were included in subsequent interviews to facilitate theory development. Memorandums were made throughout the recruitment and analysis processes in order to identify areas of interest, explore participants' perceptions, describe experiences during the interview and in relation to existing knowledge, and to provide richer data (Strauss and Corbin 1998).

Participants completed a modified version of an established frailty index at the end of the interview (Romero-Ortuno and Kenny 2012; Searle et al. 2008). This index was used for the purpose of describing characteristics of the sample recruited and to guide future sampling. The index included self-reported questions about the need for assistance in daily life, existing medical conditions, subjective health and physical activity. Binary variables were coded as per Romero-Ortuno and Kenny (2012), with 'o' indicating the absence of the deficit and ' 1 ' indicating the presence of a deficit. 
Ordinal variables were scored using a scale of o (no deficit) to 1 (deficit is maximally expressed). For example, the ordinal points for the subjective health item were: poor $=1$, fair $=0.75$, $\operatorname{good}=0.5$, very $\operatorname{good}=0.25$, excellent $=0$. Scores on the index were related to the clinical descriptors on the Clinical Frailty Scale (CFS; Rockwood et al. 2005).

The majority of participants interviewed in the early part of the research were comparatively young (less than 70 years of age) and in good health (very fit and well according to the CFS). To increase the likelihood of the final sample including a broad range of frailty experiences, potential participants in later interviews were asked about their health (e.g. 'Do you have any health conditions?') during the initial contact with the researcher. Those who stated they did not have any health conditions were not interviewed. Therefore, participants in the later interviews were comparatively older (greater than 70 years of age) with higher CFS scores (e.g. mildly and moderately frail). Interviews lasted between 40 and 180 minutes and were audio recorded for subsequent transcription.

\section{Sample characteristics}

Thirty-five older adults initially expressed interest in participating in the study. Six individuals either did not fulfil the inclusion criteria, did not express further interest after receiving additional information about the study, or (later in the recruitment process) were not interviewed because they were younger and in good health. This left a final sample of 29 participants aged 66-98 years. All participants lived in the South West of England and the sample included both community dwellers and care home residents. Based on their Frailty Index scores, the frailty status of participants varied from 'very fit' to 'moderately frail', and 'previously frail'. Participants were not directly asked about their life history, although many disclosed personal details pertaining to this. Marital status was disclosed by 25 participants $(16$ married, five widowed, two single, two divorced). The majority of the participants were retired. Table 1 details the characteristics of the sample.

\section{Data analysis}

Initial coding involved naming data line-by-line. Focused coding assigned conceptual labels to the initial themes and these were refined through repeated inspection. Axial coding involved an examination of the relationships between concepts in order to develop the emerging theory. Data collection and analysis were performed iteratively so themes and concepts were revised when novel or conflicting data were gathered. Constant comparison and reflection was performed at each stage of the research, along with scrutiny of sampling procedures, interview conduct, code creation and theory development. 
TABLE 1. Characteristics of participants

\begin{tabular}{|c|c|c|c|c|c|c|}
\hline Name & Sex & Age & Identify as frail & Frailty measure & Marital status & Profession \\
\hline Betty & Female & 82 & No, blame it on the system & Well & Widowed & Undisclosed \\
\hline Beth & Female & 84 & Yes & Managing well & Undisclosed & Office worker until married \\
\hline Caitlyn & Female & 78 & Only mobility & Moderately frail & Divorced & Professor \\
\hline Don & Male & 77 & Only a little bit & Managing well & Married & Navy \\
\hline Diana & Female & 79 & No, I fight against it & Moderately frail & Married & Doctor \\
\hline Garth & Male & 86 & No because I am not & Apparently vulnerable & Married & Solicitor \\
\hline Gemma & Female & 70 & No & Very fit & Married & Physiotherapist \\
\hline Gail & Female & 70 & No & Managing well & Widowed & School counsellor \\
\hline Ida & Female & $6_{5}$ & Not at the moment & Managing well & Single & Nurse \\
\hline Jack & Male & 88 & No & Managing well & Married & Civil servant \\
\hline Jacob & Male & 69 & No & Managing well & Married & Veterinarian \\
\hline Josh & Male & 66 & No & Apparently vulnerable & Married & Researcher \\
\hline Joanna & Female & 66 & Not particularly & Very fit & Widowed & Dietitian \\
\hline Laura & Female & 68 & Not at the moment & Well & Married & Social worker \\
\hline Lonnie & Female & $9^{8}$ & Yes & Mildly frail & Widowed & Nurse \\
\hline Mae & Female & 69 & Not at the moment no & Very fit & Married & Machinist \\
\hline Mary & Female & 83 & Not quite, most of the time I am fine & Well & Widowed & Housewife \\
\hline Maggie & Female & 83 & No, well in other ways & Apparently vulnerable & Undisclosed & Office worker \\
\hline Molly & Female & 70 & I don't feel like that at the moment & Well & Married & Nurse \\
\hline Mandy & Female & 79 & No & Managing well & Divorced & Undisclosed \\
\hline Nick & Male & 73 & I do not identify with it & Managing well & Married & Health-care educator \\
\hline Patricia & Female & 83 & Legs are only frail part & Moderately frail & Undisclosed & Housewife \\
\hline Robert & Male & 79 & Yes & Mildly frail & Married & Civil servant \\
\hline Sarah & Female & 77 & No, still have my independence & Mildly frail & Undisclosed & Researcher \\
\hline Thomas & Male & 67 & Not at the moment & Very fit & Married & Business owner \\
\hline Ted & Male & 70 & Limitations due to past injury & Temporarily frail & Married & Teacher \\
\hline Theo & Male & 68 & I don't consider myself at all & Managing well & Married & Navy/care home manager \\
\hline Todd & Male & 75 & Problems getting out and about & Moderately frail & Single & Train operator \\
\hline Walter & Male & 83 & No & Managing well & Married & Engineer \\
\hline
\end{tabular}




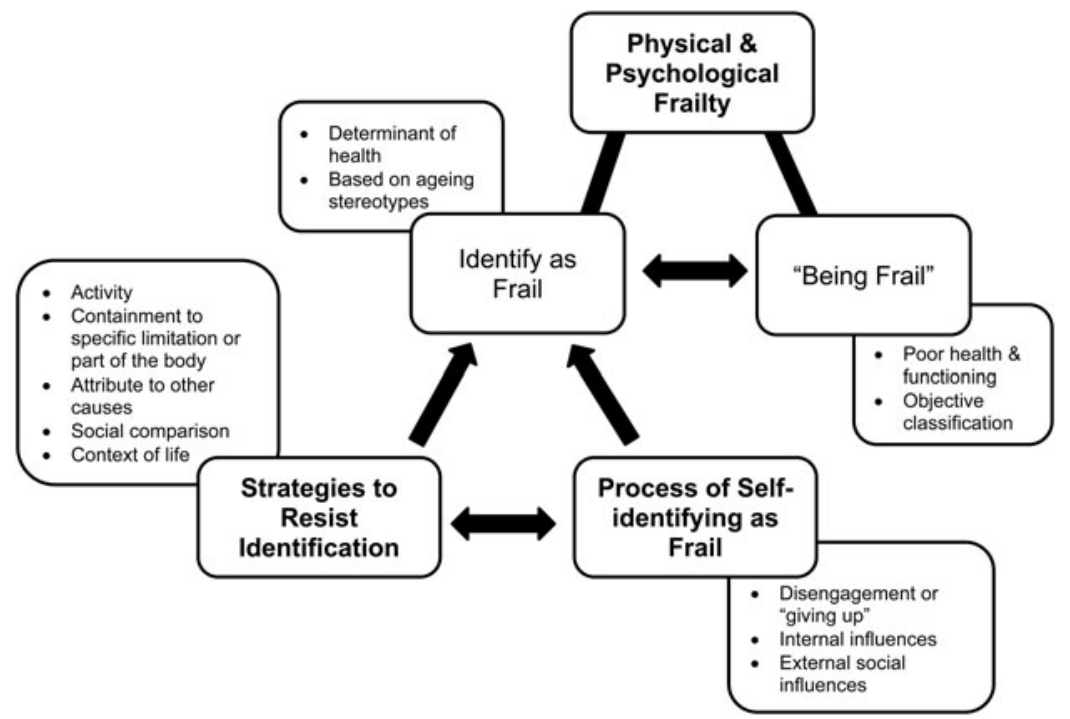

Figure 1. Model of frailty identity in older adults.

Interviews were transcribed verbatim, with pseudonyms used for participants to ensure anonymity. Coding and memo-writing were performed using NVivo 10 software (QSR International 2012) to assist with the management and organisation of the data. The first author performed the primary analysis of the findings through prolonged and persistent engagement with the data. The interpretation of data was discussed between authors and these discussions facilitated the identification of salient themes, concept refinement and theory development. Finally, lay summaries of the findings were sent for comment to the contacts and organisations who had aided recruitment; responses received were confirmatory and positive, supporting the credibility of the analysis.

\section{Results}

Analysis revealed three categories: (a) views on physical and psychological frailty; (b) the process of self-identifying as frail; and (c) strategies used to resist identification. Older adults' perceptions of frailty included the interacted concepts of 'being frail' and identifying as frail. Identifying as frail was not only informed by health but also was a believed determinant. The adoption of a frailty identity depended on resistance strategies and aspects of self-identifying (see Figure 1). The process and strategies were informed by both individual and social context. Direct quotes from participants are used in the following sections to illustrate themes within each category. 


\section{Views on physical and psychological frailty}

This category included participants' conceptualisations of frailty, both in terms of what it comprised and what it implied about the affected individual. Participants described how one could be physically and psychologically frail, showing outward symptoms or cues of frailty. Describing frail individuals, they depicted them as hunched over, grey-haired, wobbly and slow (physically and mentally). The physical characterisations referenced poor health, mobility issues (e.g. use of canes or walking frames) and other functional impairments (e.g. hearing aids). Physical decline towards dependency and death were expressed. Psychological aspects of frailty referenced negative thoughts and attitudes (e.g. thinking 'you weren't good enough to do something'), feelings of trepidation and vulnerability, inability to cope with age-related changes, and mental functioning (e.g. memory loss). These conceptualisations reflected common negative old-age stereotypes and beliefs about ageing.

However, the presence of these physical or psychological symptoms or cues did not necessarily indicate the individual actually considered themselves as frail: participants distinguished between 'being frail' and identifying as frail. 'Being frail' was related to the objective classifications of frailty and the clinical symptoms of having poor health and functional limitations, but identifying as frail incorporated the negative, and feared, views about older people as feeble, dependent and vulnerable.

Self-identifying as frail was seen as an important determinant of health outcomes above and beyond the effects of showing clinical symptoms of frailty. Participants referenced a 'cycle of decline', whereby frail people become progressively more inactive over time. Jacob, 69, explains this process:

they get a bit old age in the joints maybe a knee gets a bit sore then that's I mean pain is a big, big a cause of frailty isn't it? You begin to wonder whether you can actually walk anymore .... and you think I can't do it and you get depressed and you become frail and it's a kind of on-going cycle of decline.

Jacob described how an awareness of a physical change (e.g. sore knee) was exacerbated by thoughts about his abilities and a growing acceptance that he was physically unable. As a result, this led to depression and further physical decline. Consequences of seeing oneself as frail would become physical manifestations that furthered decline. Moreover, participants described how identifying as frail led not only to frailty but other health problems and ultimately death.

While most participants' characterisations of frailty were negative and highlighted its damage on health and wellbeing, participants also relayed experiences with older adults who willingly identified as frail, who 
'wallowed' in it, or exaggerated their limitations. This process was described by Jack:

They are probably a bit frail but ... some people say they wallow in it but I mean there are those sort of people really. They want you to feel sorry for them and they feel sorry for themselves but maybe they feel better if you ... feel sorry for them.

These individuals were said to even act in a 'frail manner' in order to secure support from other people. Perhaps, 'wallowing' is a way for people to cope with feelings or experiences of frailty.

\section{The process of self-identifying as frail}

The three participants who identified as frail believed that frailty was due to factors beyond their personal control, including age, functional limitations and health conditions. One participant described how she had accepted that she was frail due to age; Lonnie stated that, at 98 years of age, she simply accepted that she was frail. Her objective health status was not mentioned. For the other participants who identified as frail, recognition and acknowledgement of their limitations and impairments resulted in the adoption of a frailty identity. Robert, 79, had multiple health conditions and functional limitations; for him, these determined his identity as frail. When he was asked why he considered himself frail, he said:

Oh, because I can't, can't. I'm frail in every sense of the word because of my incapacity, my lack of balance, my inability to walk, to stand up, um no, no, I'm frail because I am.

He described himself as a prototype of frailty and perceived that he could not deny his obvious limitations or attribute them to other causes (e.g. a stroke or specific disease). According to these individuals, identifying as frail was an inevitable or uncontrollable issue that occurred with old age. These participants expected physical decline with increased age and selfidentification as frail was an acceptance of this natural process.

Conversely, for the majority of participants, the presence of health conditions or physical decline did not result in self-identifying as frail. Some participants did not view their limitations or health conditions as serious enough to warrant the label 'frail'. Indeed, according to biomedical definitions, they were not classified as frail. However, most of those who had multiple health deficits or classified as frail on the CFS did not identify as frail-despite readily acknowledging their health-related impairments. One clear example of participants exhibiting resistance to a frailty identity came from Diana, 79, who had cancer and Parkinson's disease and needed daily care. When asked if she considered herself frail, she responded 


\section{Krystal Warmoth et al.}

by laughing and stating 'no'. She then explained why she did not identify as frail:

because I fight against it um I mean if I let myself go and didn't arrange things and didn't do anything I could become more frail, but I have the stimulus of people around.

In this quote, Diana states how she resists identifying as frail (or 'fights against it') and even alludes to the consequences of identifying as frail. She describes resisting frailty through her individual actions and with help from others.

Most participants described the process of identifying as frail as transitional and a condition of particular circumstances. They had control over how they thought about being frail (their impairments and limitations) and how they acted (e.g. 'fights against it'). According to them, the key determinant of whether an individual adopted a frailty identity was disengagement socially or physically - and not their physical impairments or poor health. Participants described how frail people disengage or 'give up' previously enjoyed activities. Sarah, 77, was describing her understanding of what it meant to identify as frail when she said:

easily broken which means possibly if you've almost given up and it wouldn't take much to make you give up.

Patricia, 83 , reiterated Sarah's conceptualisation of the identity when she talked to frail people in her care home.

They give up. They've got one or two here that just give up. They just don't want to know. They wonder why they're on this earth. And you do try to talk to them but don't want to know.

Disengagement from physical and social activities was thought to be a stronger indication that someone self-identified as frail than health and functioning impairments alone. Ted, who was 70 years old and recently undergone surgery to regain some functioning, distinguished between being regarded as frail and being in 'frailty mode'. He was categorised as frail on the selfreported measure (CFS) before surgery, but stated that he was not in 'frailty mode', which he described as 'sitting down' and 'wasting time'. For Ted, it was disengagement behaviours that characterised a frailty identity, which were distinct from health and functioning. Furthermore, disengagement accompanied (and possibly preceded) negative psychological affect. Participants described a negative state accompanied by a sense of fear, depression and vulnerability.

Other participants described the influence of factors external to the self - labelling and stigmatisation. Jack, aged 88, had significant hearing loss and was slow to speak and walk, but had no major health conditions. 
He felt external social factors can encourage the adoption of a frailty identity - and lead to physical decline and death:

I think once you're sort of labelled frail ... there is a tendency to get worse but I don't know whether that's ... due to psychology or not but I tend to think that there are pressures on people who are frail that they the majority tend to get worse and therefore they eventually pass away I suppose or depart this life.

Diana (79, who had Parkinson's disease and a history of cancer) discussed the effects of the stigma of being frail. She said:

If you say you're frail, people will treat you differently ... if you say that you are going to see such and such a person she's now very frail. They're afraid of involving them ... yes, I think people then treat them differently. Attitude is we shan't ask you to do things.

Not only was it believed that people who self-identified as frail disengaged (or withdrew) from participation in social activities, but when others considered them frail, they were avoided and excluded from social activities. Social isolation could occur by the person being excluded because of others and their assumptions made about the older person (i.e. that they were frail and unable to participate). Diana believed that the social consequence of being excluded was very damaging, reducing their motivation for social participation and increasing their risk of further exclusion.

The above examples highlight the effects of labelling for people who already acknowledge some of the symptoms of advancing age - who are perhaps aware of their progression towards frailty. However, other participants were clear that labelling can have important influences on health even in those not showing symptoms. Several participants described situations when they had not taken notice of their own age-related conditions until other people commented on them and mentioned their age. If an individual was treated in terms of an age-related stereotype, they might take on a frailty identity. Activating ageing stereotypes could lead an individual to see their health symptoms differently - resulting in self-identification as frail. When Theo, 68, was asked if others treated him like he was frail, he said:

I don't think you should label people as being frail um no, no I wouldn't, wouldn't want to stigmatise people by saying ya know I think you're frail um know you are getting older and you can't do as much as you perhaps would like to do.

Theo acknowledged that labelling does occur but strongly opposed the assumption that older adults by definition were unable to do certain things or needed help. Frustration with such ageist attitudes was a common experience: participants described the tendency of others to perceive ageing in stereotypical terms rather than acknowledging its dynamic process with considerable individual variation. Beyond assumptions made about them because of their age, participants discussed how being labelled as frail was 


\section{Krystal Warmoth et al.}

negative and led to identification - which, in turn, could be harmful to health. Caitlyn, 78, described some of these consequences of labelling:

I think some people feel very self-conscious about it, the fact that they would seem to be frail. Some people would regard that as a, um, I think the main fear probably if you are regarding yourself as frail is you can see there lies the path to lack, a loss of independence.

Self-identifying as frail was perceived as a potential outcome of the labelling process and related to the consequences of frailty that she had described earlier in the interview: 'losing your independence'. Her understanding was that the consequences of self-identifying as frail could also occur as a result of being labelled. Notably, the path from being labelled as frail to self-identifying as frail was not regarded as inevitable; as is discussed in the following section, there was considerable resistance.

\section{Strategies to resist identification}

Most participants did not self-identify as frail, suggesting people may have the ability to resist this process, even in the presence of influences encouraging self-perceptions as frail. From the interviews with participants who did not consider themselves frail, different methods of resistance were explored. Three main strategies to resist a frailty identity were identified. First, being active (physically, mentally or socially) even at lower levels than previously, was seen as important. This strategy was not only employed by participants who had fewer health conditions or functional impairments, but by those in a more negative state of health and who could be objectively classified as frail. Common amongst the latter participants was an emphasis on current abilities and activities. Josh had multiple health conditions but did not identify as frail. He explained:

No um I am more limited than I used to be but I can still walk and cycle and swim and dig um and you ask my wife and she'll tell you I can talk the hind leg off a donkey.

Being physically able and engaging in social activity were ways to resist identifying as frail. 'Doing things' was a strategy used by several participants to 'fight against frailty'. These participants believed engaging in social activities would deter the onset or progression of frailty.

A second strategy reported by participants was relating their experiences to a particular limitation, part of the body, or attributing it to a specific, isolated, event - rather than to a more general, chronic state of ill-health. Sarah experienced functional limitations with balance and the right side of her body as a result of a stroke but did not self-identify as frail. Directive attributions like this were made by other participants who could 
be classified as frail: drawing subtle distinctions between frailty and their particular health experiences allowed acknowledgement of the reality of their illnesses or impairments while simultaneously resisting the frail identity.

The third strategy employed by participants to avoid self-identifying as frail was social comparisons with others they considered to be frailer. These participants focused on activities they could still perform that others could not. Such downward comparisons allowed participants to view themselves more positively and distinguish themselves from who they perceived to be 'worse off'. Patricia, $8_{3}$, used a wheelchair and was considered frail on the CFS. When asked about her limitations, she replied:

sometimes I wish I was a bit more able body than I am but then I got me hands and me arms and I've got me sight ... I got me hearing whereas some poor old souls ain't got that.

She described her limitations but simultaneously emphasised that she is not one of the 'poor old souls'. She used social context (other care home residents) to resist identifying as frail.

While most participants currently resisted a frailty identity and employed strategies to avoid it, there was also an acknowledgement of the perceived inevitability of adopting the identity in the future. This view was present amongst the younger participants who had comparatively better health. Such participants highlighted the preventative actions they take to delay frailty, including following a healthy diet, exercising and 'staying active'. Mae, 69, reported no health issues, but when asked if she considered herself frail said, 'not at the moment, no'. She went on to express how she was thankful not to be frail and stated it was possible in the future she might be.

Not to say in years to come but um we'll cross that bridge when we come to it and we won't, we won't sit around waiting for it to come.

In short, participants recognised that a frailty identity might be a future aspect of the self, but failed to let that prospect change their positive approach to day-to-day living.

\section{Discussion}

The aim of this qualitative study was to explore adults' perceptions of frailty and their beliefs concerning its progression and consequences. Reflecting earlier research (Grenier 2007; Puts et al. 2009), older adults in this study described frailty as a negative physical and psychological condition. The presence of illness and impairment (an objective classification as frail) did not always coincide with feelings of frailty or identifying as frail (Grenier 2006; Puts et al. 2009). Adopting a frailty identity was not regarded 


\section{Krystal Warmoth et al.}

as an inevitable consequence of frailty-related symptoms. Identification as a frail person occurred when one: (a) embodied the assumed characteristics and behaviours of the identity, such as advanced age, physical limitations and disengagement; (b) willingly assumed that label; or (c) accepted a label imposed by others. The consequences of identifying as frail were highlighted by participants, including poor health and functioning, disengagement from physical and social activities, depressive thoughts, negative affect, stigmatisation and discrimination. For the participants who did selfidentify as frail, they did so because of an issue out of their control (i.e. age or physical limitations), and they could not deny that they were frail. While for them, physical decline led to self-identification as frail, for others it did not, and triggered resistance and positive adaptation. Most participants actively resisted the identity, even those who could be classified as frail using objective criteria. They described a frailty identity as transitional and as a condition of specific circumstances (e.g. labelling and treatment from others). They used a variety of resistance strategies: (a) emphasising abilities and activity; (b) attribution to particular cause, part of the body or event; or (c) social comparisons (see Figure 1 ).

The conceptualisation of frailty varied between older adults and differed from that used in clinical contexts. The classification of frailty is often used to identify patients who are more likely to suffer adverse outcomes or unintended side-effects of interventions or medications (Clegg et al. 2013); a classification can be helpful for care-planning. However, the current interviews highlighted a discrepancy between the way frailty is operationalised in clinical settings and older adults' personal understanding. Older adults' views of frailty reflected common old-age stereotypes (see Hummert 2011) and were similarly negative (e.g. Grenier 2006; Puts et al. 2009), including psychological (thoughts of giving up and feelings of vulnerability), behavioural (disengagement) and social (stigmatisation) components. Furthermore, participants in this study discussed frailty and disablement interchangeably, in contrast to the clinical conceptualisation of it as a vulnerable state that could lead to adverse health outcomes like disability (Fried et al. 2004). Although this previous research has investigated older adults' descriptions of old age and frailty, this work explored further by questioning how older adults interact with this representation and its possible effects. Psychologically identifying as frail was regarded by older adults to have deleterious consequences for health and wellbeing. Specifically, self-identifying as frail stemmed from and could lead to disengagement from physical or social activities which, in turn, would lead to reduced physical functioning and further decline. In short, older adults in this study perceived frailty in terms of a 'cycle of decline' in which health changes can both be a cause and an outcome of self-identifying as 
frail. This study was the first to provide evidence of this relationship between older adults' beliefs regarding frailty and their health; that is, identifying as frail was not only informed by health but also was a believed determinant of it.

Furthermore, the discrepancy between the clinical understanding of frailty and the way people perceive frailty has important implications for older people's wellbeing (especially, when receiving care or support). While frailty classifications can be useful in guiding clinical care, our findings suggest that it is also important to take account of how individuals and others perceive and respond to their experiences of frailty. As participants in this study expressed, being labelled as frail - particularly against one's will - was seen as damaging to health, because it may lead to behavioural confirmation of the label. In other words, treating people as if they are frail may increase the likelihood they will self-identify as frail. When labelling occurs, unless people possess strategies to resist, their health and wellbeing may be compromised. When treating or caring for older adults, one must be aware of how their actions may impact them or how the older adult may perceive them. Further work is needed to identify which behaviours or sources may be the most harmful by encouraging older adults to self-identity as frail. For example, the act of offering assistance from a particular individual (e.g. a young stranger) may indicate to the older adult that they are viewed by them in a stereotypical way, such as being unable or even frail.

In many ways, older adults' experiences reflected those of members of other socially stigmatised groups, such as racial minorities or people with obesity (Major, Mendes and Dovidio 2013). The current participants described the readiness of others to label them as frail, often on the basis of isolated events or temporary impairments. Given the negativity of the frailty identity and its believed consequences, it was unsurprising that most participants resisted self-identifying as frail. These resistance strategies were a way to 'protect' the individual self - to affirm an individual identity (distinct from the negative frailty identity) or to articulate a preferred identity. For example, older adults' dissociation from their age group has led to better psychological wellbeing (Weiss and Lang 2009). This study explored the possible ways older adults resisted this identity and has proposed a few strategies by older adults in different conditions and contexts. However, future work exploring the ways older adults can be encouraged to employ these resistance strategies is warranted. Using this knowledge, the harmful consequences from self-identify as frail may be avoided because older adults would have positive responses to age-related changes or ill-health.

While resistance to frailty featured heavily in the discourse of participants, resistance is unlikely to be the response of everyone. Several participants 
described people they knew who readily adopted a frailty identity and used this identity to gain attention and assistance from others ('wallowing' in frailty). Readily adopting a frailty identity could damage one's health, as suggested earlier; however, it is possible that assuming a frailty identity could be beneficial to that individual. Previous research has found that individuals with chronic illnesses change or assume identities (e.g. 'the patient' or 'ill') to receive financial resources and support from others (Charmaz 1995). Disengaging from activities and showing inability may be ways of performing this identity to receive some benefits. Indeed, frailty stereotypes have been invoked for the purposes of collective action and can feature in political agendas; Grenier and Hanley (2007) showed how a frail identity can be used to challenge preconceptions about ageing and improve care and services. Therefore, if an older adult identifies and acts as 'frail', they may be more likely to attract attention, obtain support from the people around them or gain access to services. Further investigation is needed into the reasons and situations where an older person may benefit from assuming a frail identity.

A limitation of the current research is that the sample did not include any participants classified as severely frail on the CFS, or older adults with significant cognitive impairments. If such individuals were included, then different perceptions of frailty may have emerged. While the sample included individuals with diverse experiences with frailty, a large age range, varied backgrounds and a range of living situations, sampling occurred in a relatively small geographical area. Older adults with different demographics may report different experiences and understandings of frailty.

To conclude, this qualitative study examined older adults' perceptions of frailty and their beliefs concerning its progression and health consequences. From the findings, an understanding of frailty as a self-relevant identity as well as the process by which self-identification occurs and is resisted, is offered. Understanding the experiences and development of a frailty identity may inform the approaches taken to address the health and activity levels of older adults. Older adults' perceptions must be acknowledged and reflected in their care.

\section{Acknowledgements}

This report presents independent research funded by the National Institute for Health Research (NIHR) Collaboration for Leadership in Applied Health Research and Care for the South West Peninsula. The views expressed in this publication are those of the authors and not necessarily those of the National Health Service, the NIHR or the Department of Health in England. The study was approved by Peninsula College of Medicine and Dentistry Research Ethics Committee. Krystal 
Warmoth conducted analysis and interpretation of the data and drafted the manuscript. Iain Lang, Charles Abraham and Mark Tarrant conceived the study and contributed to interpretation of the data. Cassandra Phoenix helped with the design and analytic strategy of the data. Melissa K. Andrew and Ruth E. Hubbard revised the draft critically for important intellectual content. All authors contributed to and approved this version of the paper. There are no conflicts of interest.

\section{References}

Andrew, M. K., Fisk, J. D. and Rockwood, K. 2012. Psychological well-being in relation to frailty: a frailty identity crisis? International Psychogeriatrics, 24, 8, 1347-53.

Borges, L. and Menezes, R. 2011 . Definitions and markers of frailty: a systematic review of literature. Reviews in Clinical Gerontology, 2 1, 1, 67-77.

Charmaz, K. 1995. The body, identity, and self: adapting to impairment. The Sociological Quarterly, 36, 4, 657-80.

Charmaz, K. 2006. Constructing Grounded Theory: A Practical Guide Through Qualitative Analysis. Sage, London.

Clegg, A., Young, J., Iliffe, S., Rikkert, M. O. and Rockwood, K. 2013 . Frailty in elderly people. The Lancet, $\mathbf{3 8 1}, 9868,75^{2-62 .}$

Coleman, P. 1999. Identity management in later life. In Woods, R. T. (ed.), Psychological Problems of Aging: Assessment, Treatment, and Care. John Wiley and Sons, New York, 93-113.

Cotter, V. T. and Gonzalez, E. W. 20og. Self-concept in older adults: an integrative review of empirical literature. Holistic Nursing Practice, 23, 6, 335-48.

Fillit, H. and Butler, R. N. 20og. The frailty identity crisis. Journal of the American Geriatrics Society, 57, 2, 348-52.

Fried, L. P., Ferrucci, L., Darer, J., Williamson, J. D. and Anderson, G. 2004. Untangling the concepts of disability, frailty, and comorbidity: implications for improved targeting and care. Journals of Gerontology: Biological Sciences and Medical Sciences, 59B, $3,255^{-6} 3$.

Glaser, B. G. and Strauss, A. L. 1967. The Discovery of Grounded Theory: Strategies for Qualitative Research. Aldine, Chicago.

Grenier, A. 2005. The contextual and social locations of older women's experiences of disability and decline. Journal of Aging Studies, 19, 2, 131-46.

Grenier, A. 2006. The distinction between being and feeling frail: exploring emotional experiences in health and social care. Journal of Social Work Practice, 2o, 3, 299-313.

Grenier, A. 2007. Constructions of frailty in the English language, care practice and the lived experience. Ageing $\mathcal{E}$ Society, 27, 3, 425-45.

Grenier, A. and Hanley, J. 2007. Older women and 'frailty'. Current Sociology, 55, 2, $211-28$.

Heuberger, R.A. 2011. The frailty syndrome: a comprehensive review. Journal of Nutrition in Gerontology and Geriatrics, 3o, 4, 31 $5^{-68 .}$

Hummert, M. L. 2011 . Age stereotypes and aging. In Schaie, K. W. and Sherry, L. W. (eds), Handbook of the Psychology of Aging. Academic Press, San Diego, California, 249-62.

Major, B., Mendes, W. B. and Dovidio, J. F. 2013. Intergroup relations and health disparities: a social psychological perspective. Health Psychology, 32, 5, 514-24.

Morley, J. E., Perry, H. M. and Miller, D. K. 2002. Something about frailty. Journals of Gerontology: Biological Sciences and Medical Sciences, 57B, 11, 698-704. 


\section{$15^{\mathrm{OO}}$ Krystal Warmoth et al.}

Petrie, K. J., Jago, L. A. and Devcich, D. A. 2007. The role of illness perceptions in patients with medical conditions. Current Opinion in Psychiatry, 2o, 2, 163-7.

Puts, M. T. E., Lips, P. and Deeg, D. J. H. 2005. Sex differences in the risk of frailty for mortality independent of disability and chronic diseases. Journal of the American Geriatrics Society, 53, 1, 40-7.

Puts, M. T. E., Shekary, N., Widdershoven, G., Heldens, J. and Deeg, D. J. H. 2009. The meaning of frailty according to Dutch older frail and non-frail persons. Journal of Aging Studies, 23, 4, 258-66.

QSR International 2012. NVivo Io [computer software]. QSR International, Melbourne.

Rockwood, K., Song, X., MacKnight, C., Bergman, H., Hogan, D. B., McDowell, I. and Mitnitski, A. 2005. A global clinical measure of fitness and frailty in elderly people. Canadian Medical Association Journal, 173, 5, 489-95.

Romero-Ortuno, R. and Kenny, R. A. 201 2. The frailty index in Europeans: association with age and mortality. Age and Ageing, 41 , 5, 684-9.

Searle, S. D., Mitnitski, A., Gahbauer, E. A., Gill, T. M. and Rockwood, K. 2008. A standard procedure for creating a frailty index. BMC Geriatrics, 8, 24.

Strauss, A. and Corbin, J. 1998. Basics of Qualitative Research: Techniques and Procedures for Developing Grounded Theory. Sage, Thousand Oaks, California.

Weiss, D. and Lang, F. R. 2009. Thinking about my generation: adaptive effects of a dual age identity in later adulthood. Psychology and Aging, 24, 3, 729-34.

Accepted 20 April 2OI5; first published online 9 June 2015

Address for correspondence:

Krystal Warmoth,

University of Exeter Medical School,

University of Exeter,

St. Luke's Campus,

Heavitree Road,

Exeter EX $12 \mathrm{LU}, \mathrm{UK}$

E-mail: kw317@exeter.ac.uk 